\title{
La enseñanza universitaria: tablero para armar
}

\section{College education: board to build}

\author{
Carina Lion \\ https://orcid.org/0000-0002-4085-5164 \\ carinalion@gmail.com \\ Facultad de Filosofía y Letras | \\ Universidad de Buenos Aires | Argentina
}

\section{RESUMEN}

El artículo es un ensayo que recorre las transformaciones en la enseñanza universitaria y los cambios en los modos de aprender cuando hay mediación tecnológica. Desde múltiples perspectivas ofrece una suerte de mapeo de escenarios y alternativas para la toma de decisiones didácticas en relación con la inclusión de tecnologías y presenta algunas definiciones e interrogantes que serán cruciales para la institución universitaria en los próximos años. De esta manera entrecruza condiciones institucionales y políticas; escenarios contemporáneos para la construcción del conocimiento y definiciones didácticas en función de las transformaciones culturales, tecnológicas y cognitivas que se han hecho más visibles en tiempos de pandemia. Estas definiciones engloban decisiones acerca del perfil del egresado universitario en la actualidad; del curriculum y su relevancia y de los sujetos contemporáneos con sus aprendizajes emergentes.

\section{ABSTRACT}

The article is an essay that traces the transformations in university teaching and the changes in the ways of learning when there is technological mediation. From multiple perspectives, it offers a kind of mapping of scenarios and alternatives for didactic decision-making in relation to the inclusion of technologies and presents some definitions and questions that will be crucial for the university institution in the coming years. In this way it interweaves institutional and political conditions; contemporary scenarios for the construction of knowledge and didactic definitions according to the cultural, technological and cognitive transformations that have become more visible in times of pandemic. These definitions encompass decisions about the profile of today's university graduate, the curriculum and its relevance, and contemporary subjects with their emerging learning.
PALABRAS CLAVE

enseñanza universitaria, alteraciones, hackeo de plataformas experiencias inéditas, aprendizajes emergentes.

\section{KEY WORDS}

university teaching disruption, platform hacking, new experiences, emergent learning. 


\section{1- INTRODUCCIÓN}

En los últimos tiempos la enseñanza universitaria se ha visto interpelada. Por un lado, porque se han visto los límites de una didáctica clásica basada en una secuencia lineal progresiva de explicación-aplicación y verificación. Los sujetos que habitan nuestras aulas exigen otras dinámicas y nos desafían para que diseñemos propuestas diferentes (Serres, 2013). Por otro lado, las tecnologías nos invitan a deconstruir patrones, certezas y rutinas. La pandemia como punto de inflexión, nos mostró la necesidad de transformar nuestras prácticas de enseñanza. Entonces, partimos de reconocer que los escenarios contemporáneos plantean desafíos multidimensionales complejos: políticos, sociales, culturales, comunicacionales, didácticos, cognitivos y tecnológicos en los cuales hay rasgos centrales que debieran analizarse para comprender algunas tendencias en relación con las mutaciones vinculadas con los aprendizajes y los desafíos para la enseñanza universitaria. Estos escenarios se encuentran atravesados, muy profundamente, por la incertidumbre y la complejidad. Tal como señala Morin (2016) "incertidumbres y duda están vinculadas (...) nos permiten romper determinismos y reduccionismos científicos (..) hay que aprender a navegar en un océano de incertidumbres a través de archipiélagos de certeza. Habrá que enseñar principios de estrategia que permitan enfrentar los riesgos, lo inesperado y lo incierto y modificar su desarrollo en virtud de informaciones adquiridas en el camino. La incertidumbre no se elimina: se negocia con ella" (pp 32-38). En un año en que hemos transitado incertidumbres, pero también hemos podido construir algunos "archipiélagos de certezas":

\section{La pandemia como punto de inflexión, nos mostró la necesi- dad de transformar nuestras prácticas de enseñanza.}

- $\quad$ El trabajo en colectivos docentes brindó soporte y trama para hacer emerger experiencias de nuevo tipo; para compartir reflexiones y para transitar momentos de angustia, temor y soledad. 
- Los diálogos fueron sumamente necesarios para instalar conversaciones honestas, empáticas y sostenidas; para estar cerca de nuestros colegas y de nuestros estudiantes; para tejer redes de intercambio y enriquecer nuestra tarea docente.

- Muchas de las conversaciones se sostuvieron en múltiples formatos y en plataformas y redes que pudieron expandirlas; traer otras voces; construir una coralidad necesaria, amistosa y de sostén permanente.

- $\quad$ Los tiempos y formatos de la enseñanza se han visto alterados. Hubo que negociar las condiciones institucionales; comprender las condiciones para el aprendizaje (conectividad y dispositivos, pero también la trama emocional de nuestros estudiantes); reinventar las clases (Maggio, 2018); seleccionar contenidos en relación con su relevancia; priorizar tareas para la construcción de conocimiento; diseñar materiales digitales; redireccionar los esfuerzos para una retroalimentación permanente; entre otras dimensiones.

Estas certezas nos permiten definir que no podemos volver atrás como si nada hubiera sucedido. No somos los mismos como docentes; pero nuestros estudiantes tampoco lo son. Hemos atravesado esos mares de incertidumbre y los hemos "capeado". Hemos rediseñado nuestras estrategias didácticas y elaborado una agenda de nuevas preguntas; hemos construido saber didáctico en el hacer distinto; lo estamos recabando, sistematizando, documentando e interpretando para la construcción de nuevas categorías para la enseñanza universitaria tal como esbozamos a continuación.

\section{Estas certezas nos permiten definir que no podemos vol- ver atrás como si nada hubiera sucedido. No somos los mismos como docentes; pero nuestros estudiantes tampoco lo son. Hemos atravesado esos mares de incertidumbre y los hemos "capeado".}

\section{2 - SENTIDOS ALTERNATIVOS}

Hay algunas categorías incipientes que nos permiten ir armando un tablero de caminos, decisiones y experimentaciones didácticas frente a lo que se vislumbra como posibles horizontes en lo que sigue.

\subsection{Hackear las plataformas}

Los avances de la inteligencia artificial y de un contexto dataficado y digitalizado (Williamson, 2018) nos ofrecen plataformas de aprendi- 
zaje adaptativo que leen rendimiento y emociones para dar respuesta segmentada, individualizada sin necesidad de docentes que reconozcan trayectorias e interpelen los aprendizajes. La computación afectiva construye algoritmos emocionales; sumado al rendimiento académico que construye algoritmos de seguimiento estudiantil, ¿qué márgenes reales tenemos de autonomía para la toma de decisiones didácticas? En el contexto de experiencias desmaterializadas por lo digital, la fragmentación de las informaciones y la ruptura de relatos lineales nos encontramos frente a una doble fuerza motriz con un mundo y un ultramundo digital que conviven en simultáneo y a través de una suma de presencias y de experiencias (Baricco, 2019) que coexisten. Esta revolución mental que el autor analiza haciendo una maravillosa genealogía por sus huellas y fósiles ha simplificado las mediaciones y nos han traído el desafío de hacerlas visibles. Un recorrido por los últimos años de la informática (desde el DOS hasta hoy), da cuenta de que se han borrado las mediaciones al hacer más simple el acceso a la tecnología a través de aplicaciones que se encuentran a un "click" de distancia. Esta invisibilización de las mediaciones; que de alguna manera superficializa las experiencias (Baricco, 2019) brinda un acceso casi "infinito" a grandes volúmenes de información y aplicaciones que, en realidad, responden a intereses corporativos y sociales que dotan de complejidad este análisis.

\section{Esto implica, no reducir las propuestas ni las tareas a las he- rramientas que estas plataformas proponen sino, por el con- trario, definir cuál es la mejor clase que puedo realizar en la virtualidad y "domesticar" las plataformas para que estas experiencias resulten vivencias únicas y no algoritmizadas ni replicables en escala.}

Series, videojuegos y otros objetos de la cultura ofrecen distopías que se asemejan a la realidad; cada vez hay menos espacio entre la imaginación del futuro y el futuro; entre los escenarios retrotópicos (Bauman, 2019), utópicos y distópicos. En una era de alteraciones y discrepancias en la que casi todo puede pasar; una era de crisis instrumentales persistentes, estamos invitados a promover una cultura de diálogo para reconstruir el tejido social (Bauman, op. Cit.). El diálogo nos recuerda que no somos espectadores ni observadores sino participantes activos en estos escenarios que se pueden transformar en esperanzadores si encontramos intersticios entre una autonomía ilusoria y controlada y la sensación de un progreso indefinido. Son estos diálogos y este sentido de agencia (Bruner, 1997) los que nos llevan a hackear las plataformas; darle sentido desde nuestros propósitos; intervenirlas desde la didáctica. Esto implica, no reducir las propuestas ni las tareas a las herramientas que estas plataformas proponen sino, 
por el contrario, definir cuál es la mejor clase que puedo realizar en la virtualidad y "domesticar" las plataformas para que estas experiencias resulten vivencias únicas y no algoritmizadas ni replicables en escala.

\subsection{Habitar un "doble trackeo".}

Escuchamos cada vez con más fuerza las ideas de prácticas híbridas que combinan presencialidad y virtualidad; sincronía y asincronía. Creemos firmemente en que ¿Cómo realizar un seguimiento de los aprendizajes de nuestros estudiantes en la bimodalidad o en prácticas híbridizadas?

\section{Entendemos que cuando nos referimos a hibridaciones es- tamos dando cuenta de combinaciones de formatos; de es- trategias didácticas; de alternancias entre la virtualidad y la presencialidad; de plataformas; es decir, que son múltiples y variadas estas combinaciones y no se reducen binariamente a presencialidad y virtualidad.}

Entendemos que cuando nos referimos a hibridaciones estamos dando cuenta de combinaciones de formatos; de estrategias didácticas; de alternancias entre la virtualidad y la presencialidad; de plataformas; es decir, que son múltiples y variadas estas combinaciones y no se reducen binariamente a presencialidad y virtualidad. Esto aporta complejidad al seguimiento de trayectorias estudiantiles que transcurren en simultáneo en espacios, tiempos, dispositivos diversos y variados y que exigen algunas decisiones:

- Qué condiciones políticas e institucionales son necesarias para dar viabilidad a una continuidad pedagógica inclusiva y retentiva.

- $\quad$ Qué vale la pena de ser enseñado con mediación tecnológica (sincrónica y asincrónica) y qué experiencias resultan vitales en la presencialidad (entendiendo que siempre hay presencia, solo que se manifiesta de otra manera);

- Qué contenidos circulan por las redes y pueden ser de expansión y de enriquecimiento (pero están a un "click" de distancia y ameritan una buena curaduría de contenidos); qué contenidos son centrales.

- Optar por profundidad y no por extensión. Situar las actividades para que revistan sentido en relación con las trayectorias e intereses de nuestros/as estudiantes y no solo porque "son temas que hay que enseñar"; entender las actividades situadas y contextualizadas en relación 
con el nivel educativo al que hacen referencia, a la especificidad disciplinar; a los recorridos estudiantiles y teniendo en cuenta que los tiempos de aprendizaje son diversos y variados.

- Combinar lo cognitivo con lo emocional (cuando el sostén del vínculo es más necesario que nunca en estos tiempos.

- $\quad$ Realizar mapeos de seguimiento con indicadores que den cuenta de cómo van nuestros estudiantes.

\subsection{Diseñar experiencias inéditas.}

Baricco (2019) señala con perspicacia que las tecnologías han eliminado ciertas mediaciones y las élites que sostenían dichas mediaciones (sacerdotes, docentes), pero han instalado otro tipo de mediación más simple (por lo menos en la superficie). Herramientas, entornos y aplicaciones buscan hacernos "la vida más sencilla"; a un click de distancia. Frente a la tendencia de simplificación tecnológica creemos que un desafío es cierta sofisticación cognitiva para la construcción de conocimiento que pueda ir "más allá de la información dada" (Bruner, 1997). Ahora bien, ¿cuál es el alcance de esta sofisticación? Si algo vimos que trajo la pandemia es sobrecarga de tarea; saturación cognitiva; tiempos intensos de trabajo (para docentes y estudiantes), poco margen para tiempo libre, de descanso, de ocio. La "activitis" (Lion, 2020b) mostró que estábamos saturando con muchísimas actividades, no siempre con sentido pedagógico.

Para dar lugar a la diversidad de aprendizajes; necesitamos el diseño de clases inclusivas; que ofrezcan anclajes a las trayectorias diversas; flexibles en tiempos y recorridos; a lenguajes expresivos múltiples (Eisner, 1998) en consonancia con la multialfabetización tanto en las estrategias de enseñanza como en las de evaluación.

\section{Diseñar propuestas de enseñanza inéditas, no previsibles, creativas que den lugar a experiencias de nuevo tipo en las que haya interfaces variadas y relevantes (entre la institu- ción educativa y su contexto; entre la enseñanza, el apren- dizaje y la evaluación; entre contenidos desafiantes e invi- taciones a pensar "fuera de la caja"; entre relatos simples y transmediáticos; entre producciones significativas y el mun- do del trabajo, etc.).}

Diseñar propuestas de enseñanza inéditas, no previsibles, creativas que den lugar a experiencias de nuevo tipo en las que haya interfaces variadas y relevantes (entre la institución educativa y su contexto; en- 
tre la enseñanza, el aprendizaje y la evaluación; entre contenidos desafiantes e invitaciones a pensar "fuera de la caja"; entre relatos simples y transmediáticos; entre producciones significativas y el mundo del trabajo, etc.). Creemos que se trata de ayudar a hacer visible el pensamiento, los procesos, las capacidades, las mediaciones. Tal como menciona Barbero (2002), el lenguaje como mediación ofrece una textura dialógica y simbólica. Es decir, ayudar a nuestros estudiantes a visibilizar lo que la informática ha invisibilizado estos últimos años al crear interfases sencillas, accesibles y cercanas a nuestra comprensión. Esto exige trabajar con procesos metacognitivos que den cuenta de qué acciones realizan cuando interactúan con plataformas, aplicaciones, herramientas tecnológicas (Lion, 2020a)

Se trata de:

- Generar prácticas disruptivas, multiexpresivas, espiraladas que inviten al co-diseño junto con los/as estudiantes; que inviten a nuevas formas de repensar la enseñanza en consistencia con las transformaciones en los cambios de aprender y de construir conocimiento en los escenarios digitales contemporáneos.

- Plantear problemas reales y relevantes que no puedan resolverse a un "click" de distancia; promover experiencias valiosas que dejen huella en el largo plazo: aprendizajes perdurables y transferencia lejana.

- Diseñar arquitecturas "móviles", flexibles comprendiendo la pluricontextualización de los aprendizajes que hibridan formatos, entornos, tiempos y espacios.

- Promover el fortalecimiento de comunidades y redes de práctica; con vínculos fuertes y apoyos entre pares.

- Evitar los reduccionismos: de enseñanza a explicación; de aprendizaje a rendimiento; de evaluación a acreditación; la propuesta didáctica a un repositorio de tareas y recursos; la multidimensionalidad a respuestas simples. Las reducciones tientan, pero no nos permiten transitar la incertidumbre y aprender de ella.

\section{TABLERO PARA ARMAR}

Tal como expresábamos en el inicio, volver atrás como si nada de esto hubiera sucedido resultaría ingenuo y empobrecedor. No obstante, reducir lo aprendido a una hibridación o clase invertida también lo sería. 
Creemos que el tablero para armar es más complejo. Sus dimensiones refieren a lo institucional, lo comunicacional, lo didáctico, lo cognitivo, lo emocional, lo epistemológico, lo cultural y por supuesto lo político.

\section{Creemos que el tablero para armar es más complejo. Sus dimensiones refieren a lo institucional, lo comunicacional, lo didáctico, lo cognitivo, lo emocional, lo epistemológico, lo cultural y por supuesto lo político.}

Ofrecemos, por tanto, algunas preguntas e ideas que permitan discutir en la comunidad docente cuáles podrán ser los próximos pasos.

La ruptura de la linealidad ha quedado ya hace tiempo demostrada en los cambios en la comunicación (fragmentada, segmentada y multiplataforma), en la extrema hiperpersonalización (las tecnologías van en esa línea a partir de la Inteligencia Artificial e Internet de las cosas) y en la enseñanza. ¿Cuáles serán las alternativas para generar propuestas de enseñanza y alterar la secuencia lineal progresiva? ¿Cómo serán los aprendizajes en los próximos años? ¿Qué indicadores de seguimiento serán necesarios para dar cuenta de trayectorias cada vez más heterogéneas? ¿Qué contenido resultará relevante y será prioritario construir que no está a un click de distancia? ¿Qué diálogos tendremos que construir con la comunidad educativa en su conjunto? ¿Qué condiciones institucionales serán necesarias para habilitar cambios profundos en las próximas décadas? ¿Cuáles deberán ser decisiones y definiciones políticas que acorten brechas de todo tipo y promuevan una inclusión auténtica en la permanencia y egreso universitarios?

Creemos que será necesario:

- Diseñar clases con formatos diferentes. Algunas invertidas, otras lúdicas, otras basadas en resolución de problemas y casos reales o verosímiles, otras que ofrezcan la ideación de proyectos relevantes o el prototipado de alguna solución (a modo de hackaton creativo). La creación didáctica no puede ser estándar. Puede sorprender, invitar al estudiantado a ser parte de su diseño; debe promover huellas que perduren, aprendizajes profundos y a largo plazo.

- Volver a discutir los perfiles profesionales y académicos. La inserción profesional es dinámica y por ende, los vínculos entre la universidad y la sociedad deben revisitarse. ¿Qué competencias serán las necesarias? ¿Qué exige el mundo del trabajo y qué prevemos pueda exigir en los próximos años justamente para no profundizar la brecha de expulsiones (Sassen, 2015)? 
- El curriculum. La pandemia nos obligó a elegir y priorizar. Este es un ejercicio pendiente en muchas carreras universitarias. El enciclopedismo y los grandes inventarios de contenidos siguen vigentes en espacios y cátedras de la universidad. Hay muchos contenidos hoy presentes en las redes; cómo generar procesos de construcción de conocimiento relevante frente a la abundancia de estos contenidos. Small data frente a Big data.

- $\quad$ Las trayectorias. Hemos aprendido que para que haya aprendizaje auténtico, tenemos que generar condiciones para que este aprendizaje tenga lugar. Condiciones que son emocionales, cognitivas, de acceso; es decir que implican una trama multidimensional. Hemos aprendido, además, que es vital realizar un seguimiento de los aprendizajes a través de una construcción de indicadores que hagan visible estos procesos que son psicológicos para poder trazar trayectorias que permitan un seguimiento más preciso de cada estudiante (seguimiento, retroalimentación oportuna y certera; evaluación permanente). Esta elaboración puede ser colectiva, pero será un ejercicio inevitable en este tablero para armar. La gamificación puede ser una alternativa valiosa para este rediseño (Lion y Perosi, 2019).

- Definiciones políticas e institucionales. Acortar brechas que son de acceso y de apropiación; redefinir las condiciones de trabajo docente para habilitar procesos creativos, inventivos e inspiradores de nuevas prácticas. Las estructuras de cátedras, en ocasiones, ponen trabas a la innovación. Habrá que repensar los tiempos y los espacios. Incorporar las mejores prácticas que podemos diseñar para la virtualidad y para la presencialidad. Proponer experiencias que dejen huella y, sobre todo, que inviten a tener un futuro inclusivo, democrático, participativo y transformador. 


\section{BIBLIOGRAFIA}

Baricco, A. (2019). “The Game”. Buenos Aires: Anagrama.

Bauman, Z. (2019). “Retrotopía”. Buenos Aires: Paidós

Bruner J. (1997). “La educación: puerta de la cultura”. Madrid: Visor

Eisner, E. (1998). “Cognición y curriculum”. Buenos Aires: Amorrortu.

Lion, C. Comp. (2020a). "Aprendizaje y tecnologías. Habilidades del presente, proyecciones de futuro". Buenos Aires: Novedades educativas.

Lion, Carina (2020b). “Listado de aprendizajes para la post pandemia”, en Revista 12ntes, ISSN 1852-6497 Número 54 - Diciembre 2020 Revista Digital 12(ntes) Sin Distancias

Lion, Carina y Perosi, Verónica (comp.) (2019). “Didácticas Lúdicas con videojuegos educativos. Escenarios y horizontes alternativos para enseñar y aprender". Editorial Novedades Educativas, Buenos Aires. ISBN Nro. 978-987-538-678-5

Maggio, M. (2018). “Reinventar la clase en la universidad”. Buenos Aires: Paidós.

Martin Barbero, J. (2002). "La educación desde la comunicación". Buenos Aires: Norma.

Morin, E. (2016). "Enseñar a vivir. Manifiesto para cambiar la educación". Barcelona: Paidós.

Sassen, S. (2015). "Expulsiones. Brutalidad y complejidad en la economía global". Buenos Aires: Katz Editores.

Williamson, B. (2018). "Big data en educación: el futuro digital del aprendizaje, la política y la práctica”. Madrid: Morata. 